\title{
Diophantine properties of numbers related to Catalan's constant
}

\author{
Tanguy Rivoal and Wadim Zudilin
}

\section{Introduction}

This article deals with Dirichlet's beta function which is defined by

$$
\beta(s):=\sum_{n=0}^{\infty} \frac{(-1)^{n}}{(2 n+1)^{s}}
$$

for any complex $s$ such that $\operatorname{Re}(s)>0$. Euler showed that, for odd $s, \beta(s)$ is a rational multiple of $\pi^{s}$, namely

$$
\beta(2 n+1)=\frac{(-1)^{n} E_{2 n}}{2^{2 n+2}(2 n) !} \pi^{2 n+1} \quad \text { for } n=0,1,2, \ldots
$$

Here the integers $E_{2 n}$ are the Euler numbers, whose exponential-generating function is given by the formula

$$
\frac{1}{\cosh (z)}=\sum_{k=0}^{\infty} \frac{E_{k}}{k !} z^{k}
$$

Therefore, Lindemann's theorem " $\pi$ is transcendental over $\mathbb{Q}$ " implies that the odd beta values $\beta(2 n+1)$ are transcendental over $\mathbb{Q}$.

For even $s$, no Euler-type formula is known, and no method has yet been found for determining the arithmetic nature of even beta values. Thus, the situation for the beta function appears to be the same as the situation for the Riemann zeta function

$$
\zeta(s):=\sum_{n=1}^{\infty} \frac{1}{n^{s}}
$$

This is not completely true since the equivalent of Apéry's theorem " $\zeta(3) \notin$ $\mathbb{Q}$ " [Ap] for Catalan's constant

$$
G:=\sum_{n=0}^{\infty} \frac{(-1)^{n}}{(2 n+1)^{2}}=\beta(2)
$$


has not yet been proved.

At the end of the last century, in [Ri1] (see also [BR]), a method was introduced for tackling the problem of determining the arithmetical nature of odd zeta values :

Theorem. For any odd $a \geq 3$, the dimension of the $\mathbb{Q}$-vector space spanned by $1, \zeta(3), \zeta(5), \ldots, \zeta(a)$ is greater than $\frac{1}{3} \log (a)$.

It follows that infinitely many odd zeta values are irrational. The search of new irrational odd zeta values was started in [Ri2] "At least one of the nine numbers $\zeta(5), \zeta(7), \ldots, \zeta(21)$ is irrational" and improved in [Zu2], [Zu3] :

Theorem. At least one of the numbers $\zeta(5), \zeta(7), \zeta(9), \zeta(11)$ is irrational.

We refer the interested reader to [Zu1] for similar results. Our aim is to translate both theorems above into the context of the beta function. We will prove the following two results.

Theorem 1. Let $a$ be an even integer $\geq 2$ and let $\delta(a)$ denote the dimension of the $\mathbb{Q}$-vector space spanned by $1, \beta(2), \beta(4), \ldots, \beta(a)$. Then

$$
\delta(a) \geq \frac{1+\mathrm{o}(1)}{2+\log (2)} \log (a) \quad \text { as } a \rightarrow \infty .
$$

Moreover, there exists a computable constant $c_{0}>0$ such that $\delta(a)>$ $c_{0} \log (a)$ for any even integer $a$.

Theorem 2. At least one of the seven numbers

$$
\beta(2), \beta(4), \beta(6), \beta(8), \beta(10), \beta(12), \beta(14)
$$

is irrational.

\section{The hypergeometric construction}

Let $q$ be an integer $\geq 4$ and let $\eta=\left(\eta_{0}, \eta_{1}, \ldots, \eta_{q}\right)$ be a family of $q+1$ integral parameters satisfying the conditions

$$
0<\eta_{j}<\frac{1}{2} \eta_{0} \quad \text { for } j=1, \ldots, q \quad \text { and } \quad \eta_{1}+\eta_{2}+\cdots+\eta_{q} \leq \frac{q-1}{2} \eta_{0} .
$$

For any non-negative integer $n$ consider the tuple $\underline{h}=\left(h_{0}, h_{1}, \ldots, h_{q}\right)$ given by the formulae

$$
h_{0}=\eta_{0} n+1, \quad h_{j}=\eta_{j} n+\frac{1}{2} \quad \text { for } j=1, \ldots, q-1, \quad \text { and } \quad h_{q}=\eta_{q} n+1 .
$$


Both proofs are based on a detailed study of the following hypergeometric series

$$
\begin{aligned}
S_{n}(z)= & S_{n, q, \underline{\underline{\eta}}}(z) \\
:=\gamma_{n} & \cdot \frac{\Gamma\left(1+h_{0}\right) \prod_{j=1}^{q} \Gamma\left(h_{j}\right)}{\prod_{j=1}^{q} \Gamma\left(1+h_{0}-h_{j}\right)} \\
& \quad{ }_{q+2} F_{q+1}\left(\begin{array}{cc}
5 h_{0}, 1+\frac{1}{2} h_{0}, \quad h_{1}, \quad \ldots, \quad h_{q} \\
\frac{1}{2} h_{0}, \quad 1+h_{0}-h_{1}, \ldots, 1+h_{0}-h_{q}
\end{array} \mid z^{-1}\right)_{(5)} \\
= & \gamma_{n} \cdot \sum_{t=0}^{\infty}\left(2 t+h_{0}\right) \frac{\Gamma\left(t+h_{0}\right) \prod_{j=1}^{q} \Gamma\left(t+h_{j}\right)}{\Gamma(t+1) \prod_{j=1}^{q} \Gamma\left(t+1+h_{0}-h_{j}\right)} z^{-t},
\end{aligned}
$$

which, by the conditions (3) on the parameters $\underline{\eta}$, is convergent for any complex $z,|z| \geq 1$. The factor

$$
\gamma_{n}=\gamma_{n, q, \underline{\eta}}:=\frac{\prod_{j=1}^{q-1}\left(h_{0}-2 h_{j}\right) !}{\left(h_{q}-1\right) !^{2}}=\frac{\prod_{j=1}^{q-1}\left(\left(\eta_{0}-2 \eta_{j}\right) n\right) !}{\left(\eta_{q} n\right) !^{2}}
$$

that appears in the definition of $S_{n}(z)$ corresponds to an arithmetic normalization of the series.

There is an alternative "Pochhammer representation" of (6),

$$
S_{n}(z)=\gamma_{n} \cdot \sum_{t=0}^{\infty}\left(2 t+h_{0}\right) \frac{(t+1)_{h_{q}-1}\left(t+1+h_{0}-h_{q}\right)_{h_{q}-1}}{\prod_{j=1}^{q-1}\left(t+h_{j}\right)_{1+h_{0}-2 h_{j}}} z^{-t},
$$

where $(a)_{l}:=\Gamma(a+l) / \Gamma(a)=a(a+1) \cdots(a+l-1)$ for $l \in \mathbb{N}$ denotes Pochhammer's symbol, which is very similar to the one introduced in [Ri1] and $[\mathrm{BR}]$ in the study of odd zeta values.

The key point is that the series in (5) is of a special kind. Namely it is a well-poised hypergeometric series in the terminology of [Bai], [Sl]: the sum of corresponding top and bottom parameters in (5) is invariant,

$$
h_{0}+1=\left(1+\frac{1}{2} h_{0}\right)+\frac{1}{2} h_{0}=h_{1}+\left(1+h_{0}-h_{1}\right)=\cdots=h_{q}+\left(1+h_{0}-h_{q}\right) .
$$

The expression (5) gives rise to the following Euler-Beukers-type integral representation of $S_{n}(z)$.

Lemma 1. The formula

$$
\begin{aligned}
S_{n}(z) & =\frac{\Gamma\left(1+h_{0}\right)}{\Gamma\left(h_{q}\right)^{2} \Gamma\left(1+h_{0}-2 h_{q}\right)} \\
& \times z^{h_{0}} \int_{[0,1]^{q}} \frac{\prod_{j=1}^{q} t_{j}^{h_{j}-1}\left(1-t_{j}\right)^{h_{0}-2 h_{j}}}{\left(z-t_{1} t_{2} \cdots t_{q}\right)^{h_{0}}} \frac{z+t_{1} t_{2} \cdots t_{q}}{z-t_{1} t_{2} \cdots t_{q}} \mathrm{~d} t_{1} \mathrm{~d} t_{2} \cdots \mathrm{d} t_{q}
\end{aligned}
$$

holds for any $z \in \mathbb{C},|z| \geq 1$. 
Proof. Using Section 4.1 of [Sl], we can derive the following integral representation for all complex $z$ such that $|z|>1$ :

$$
\begin{aligned}
S_{n}(z) & =\frac{\Gamma\left(1+h_{0}\right)}{\Gamma\left(h_{q}\right)^{2} \Gamma\left(1+h_{0}-2 h_{q}\right)} \\
& \times \int_{[0,1]^{q}} \prod_{j=1}^{q} t_{j}^{h_{j}-1}\left(1-t_{j}\right)^{h_{0}-2 h_{j}}{ }_{2} F_{1}\left(\begin{array}{c}
2 h_{0}, 1+\frac{1}{2} h_{0} \\
\frac{1}{2} h_{0}
\end{array} \mid t_{1} \cdots t_{q} z^{-1}\right) \mathrm{d} t_{1} \cdots \mathrm{d} t_{q} .
\end{aligned}
$$

Formula (1.5.21) in [Sl] implies that, for $|t|<1$,

$$
{ }_{2} F_{1}\left(\begin{array}{c|c}
2 h_{0}, 1+\frac{1}{2} h_{0} & t \\
\frac{1}{2} h_{0} &
\end{array}\right)=\frac{1+t}{(1-t)^{h_{0}+1}}
$$

and the desired integral formula follows for $|z|>1$. In the case $|z|=1$, mutatis mutandis, the proof is the same as the one of Lemma 2 in [BR].

The beta values are particular cases as specialisations of the Lerch function

$$
\Phi_{s}(z)=\Phi\left(s, \frac{1}{2} ; z\right):=\sum_{k=0}^{\infty} \frac{z^{k}}{(k+1 / 2)^{s}}
$$

for complex $z,|z| \leq 1$, and $s, \operatorname{Re}(s)>1$, since

$$
\beta(s)=2^{s} \Phi_{s}(-1) \quad \text { for } s \in \mathbb{C}, \operatorname{Re}(s)>0 .
$$

\section{The structure of $S_{n, q, \eta}(z)$}

We need to decompose $S_{n, q, \eta}(-1)$ as a linear form in 1 and even beta values only. The following Lemma 2 is almost the same as the corresponding one in $[\mathrm{BR}]$ and we only include a sketch of the proof. We would like to emphasise the fact that it is essentially the well-poisedness of the series that enables us to produce this parity-dependant behaviour when $z= \pm 1$.

Consider the rational function summand in (7) or (6)

$$
\begin{aligned}
R_{n}(t)=R_{n, q, \underline{\eta}}(t) & :=\gamma_{n} \cdot\left(2 t+h_{0}\right) \frac{(t+1)_{h_{q}-1}\left(t+1+h_{0}-h_{q}\right)_{h_{q}-1}}{\prod_{j=1}^{q-1}\left(t+h_{j}\right)_{1+h_{0}-2 h_{j}}} \\
& =\gamma_{n} \cdot\left(2 t+h_{0}\right) \frac{\prod_{j=0}^{q} \Gamma\left(t+h_{j}\right)}{\prod_{j=0}^{q} \Gamma\left(t+1+h_{0}-h_{j}\right)}
\end{aligned}
$$

and define the rational numbers $c_{k j}=c_{k, j, n, q, \underline{\eta}}$, where $j=1, \ldots, q-1$ and $k=h_{j}+\frac{1}{2}, \ldots, h_{0}-h_{j}+\frac{1}{2}$, as the coefficients in the partial-fraction decomposition

$$
R_{n}(t)=\sum_{j=1}^{q-1} \sum_{k=h_{j}+\frac{1}{2}}^{h_{0}-h_{j}+\frac{1}{2}} \frac{c_{k j}}{\left(t+k-\frac{1}{2}\right)^{j}} .
$$


Using this notation, we define the polynomials

$$
\begin{aligned}
& P_{j n}(z)=P_{j, n, q, \underline{\underline{\eta}}}(z):=\sum_{k=h_{j}+\frac{1}{2}}^{h_{0}-h_{j}+\frac{1}{2}} c_{k j} z^{k-1} \quad \text { for } j=1, \ldots, q-1, \\
& P_{0 n}(z)=P_{0, n, q, \underline{\underline{\eta}}}(z):=\sum_{j=1}^{q-1} \sum_{k=h_{j}+\frac{1}{2}}^{h_{0}-h_{j}+\frac{1}{2}} c_{k j} \sum_{l=1}^{k-h_{q}} \frac{z^{k-l}}{(l-1 / 2)^{j}},
\end{aligned}
$$

which belong to $\mathbb{Q}[z]$ (for $k<h_{q}$ in $(12)$, we mean $\sum_{l=1}^{k-h_{q}}:=-\sum_{l=k-h_{q}}^{-1}$, and the sum is 0 if $k=h_{q}$ ).

Remark. Following the usual procedure, we can show that

$$
c_{k j}=D_{q-1-j}\left(R_{n}(t)\left(t+k-\frac{1}{2}\right)^{q-1}\right)_{\mid t=-k+\frac{1}{2}} \in \mathbb{Q},
$$

where

$$
D_{\lambda}:=\frac{1}{\lambda !}\left(\frac{\mathrm{d}}{\mathrm{d} t}\right)^{\lambda}, \quad \lambda=0,1,2, \ldots,
$$

for the numbers $c_{k j}, k=h_{j}+\frac{1}{2}, \ldots, h_{0}-h_{j}+\frac{1}{2}$ and $j=1, \ldots, q-1$.

Lemma 2. For all complex $z,|z| \geq 1$,

$$
S_{n}(z)=\sum_{j=1}^{q-1} P_{j n}(z) \Phi_{j}\left(z^{-1}\right)-P_{0 n}(z) .
$$

Moreover, if $q$ is even then for any odd $j \geq 1, P_{j n}(-1)=0$ and hence

$$
S_{n}(-1)=\sum_{l=1}^{q / 2-1} P_{2 l, n}(-1) \cdot 2^{-2 l} \beta(2 l)-P_{0 n}(-1)
$$

is a linear form in 1 and the even beta values $\beta(2), \beta(4), \ldots, \beta(q-2)$.

Proof. Using the decomposition (10) and the fact that the rational function (9) has zeros at the points $t=-1,-2, \ldots, 1-h_{q}$, we obtain

$$
\begin{aligned}
S_{n}(z) & =\sum_{t=0}^{\infty} R_{n}(t) z^{-t}=\sum_{t=1-h_{q}}^{\infty} R_{n}(t) z^{-t} \\
& =\sum_{j=1}^{q-1} \sum_{k=h_{j}+\frac{1}{2}}^{h_{0}-h_{j}+\frac{1}{2}} c_{k j} z^{k-1} \sum_{t=1-h_{q}}^{\infty} \frac{z^{-(t+k-1)}}{\left(t+k-\frac{1}{2}\right)^{j}} \\
& =\sum_{j=1}^{q-1} \sum_{k=h_{j}+\frac{1}{2}}^{h_{0}-h_{j}+\frac{1}{2}} c_{k j} z^{k-1}\left(\sum_{l=1}^{\infty}-\sum_{l=1}^{k-h_{q}}\right) \frac{z^{-l+1}}{\left(l-\frac{1}{2}\right)^{j}}
\end{aligned}
$$


which gives the desired representation (15).

Further, the definition (9) gives that

$$
R_{n}\left(-t-h_{0}\right)=-(-1)^{h_{0}(q-1)} R_{n}(t),
$$

hence $c_{h_{0}-k+1, j}=(-1)^{h_{0}(q-1)+j-1} c_{k, j}$ for $k=h_{j}+\frac{1}{2}, \ldots, h_{0}-h_{j}+\frac{1}{2}$ and $j=1, \ldots, q-1$. Thus, as in Lemmas 1 of [Ri1], [Ri2], [BR] it follows that the polynomials $P_{j n}(z)$ are "士-reciprocal":

$$
z^{h_{0}-1} P_{j n}\left(z^{-1}\right)=(-1)^{h_{0}(q-1)+j-1} P_{j n}(z), \quad j=1, \ldots, q-1,
$$

and the substitution $z=-1$ gives us to the relations

$$
P_{j n}(-1)=(-1)^{h_{0} q+j} P_{j n}(-1), \quad j=1, \ldots, q-1,
$$

that is $P_{j n}(-1)=0$ for odd $j, 1 \leq j \leq q-1$, provided that $q$ is even.

\section{Asymptotic behaviour of the series}

In this paragraph, we determine the asymptotics of $S_{n, q, \eta}(-1)$.

Lemma 3. As $n \rightarrow \infty$, the limit of $\left|S_{n}(-1)\right|^{1 / n}$ exists and is equal to

$$
\varphi=\varphi_{q, \underline{\eta}}:=\frac{\eta_{0}^{\eta_{0}} \eta_{q}^{-2 \eta_{q}}}{\left(\eta_{0}-2 \eta_{q}\right)^{\eta_{0}-2 \eta_{q}}} \cdot \max _{\underline{t} \in[0,1]^{q}} \frac{\prod_{j=1}^{q} t_{j}^{\eta_{j}}\left(1-t_{j}\right)^{\eta_{0}-2 \eta_{j}}}{\left(1+t_{1} t_{2} \cdots t_{q}\right)^{\eta_{0}}} .
$$

In particular, $\varphi_{q, \underline{\eta}} \neq 0$.

Proof. Using the integral representation (8) for $S_{n}(-1)$ together with the correspondence (4) between the parameters $\eta$ and $\underline{h}$, the existence and the theoretical value of the limit follow easily from the Laplace method and arguments such as those used in the first proof of Lemma 3 in $[\mathrm{BR}]$. The non-vanishing of $\varphi_{q, \underline{\underline{\eta}}}$ is clear from its definition.

Remark. In fact, the maximum in (17) is achieved at a point $\underline{t}=\left(t_{1}, \ldots, t_{q}\right) \in$ ] $0,1\left[^{q}\right.$ that can be calculate as a function of some root $\left.t_{0}=t_{0}(\eta) \in\right] 0,1[$ of the polynomial

$$
Q(t)=Q_{q, \underline{\eta}}(t):=t \prod_{j=1}^{q}\left(\left(\eta_{0}-\eta_{j}\right)-\eta_{j} t\right)-\prod_{j=1}^{q}\left(\eta_{j}-\left(\eta_{0}-\eta_{j}\right) t\right) .
$$

Indeed, setting

$$
F(\underline{t})=\log \left(\frac{\prod_{j=1}^{q} t_{j}^{\eta_{j}}\left(1-t_{j}\right)^{\eta_{0}-2 \eta_{j}}}{\left(1+t_{1} t_{2} \cdots t_{q}\right)^{\eta_{0}}}\right)
$$


we see that the extrema in (17) are attained at points satisfying the conditions

$$
0=\frac{\partial F}{\partial t_{j}}=\frac{1}{t_{j}}\left(\eta_{j}-\frac{\eta_{0}-2 \eta_{j}}{1-t_{j}} t_{j}-\eta_{0} \frac{t_{1} t_{2} \cdots t_{q}}{1+t_{1} t_{2} \cdots t_{q}}\right), \quad j=1,2, \ldots, q .
$$

Set $t_{0}:=t_{1} t_{2} \cdots t_{q}$; then by (19) we obtain

$$
\eta_{j}-\frac{\eta_{0}-2 \eta_{j}}{1-t_{j}} t_{j}=\frac{\eta_{0} t_{0}}{1+t_{0}}, \quad j=1,2, \ldots, q,
$$

that yields

$$
t_{j}=\frac{\eta_{j}-\eta_{0} t_{0} /\left(1+t_{0}\right)}{\eta_{0}-\eta_{j}-\eta_{0} t_{0} /\left(1+t_{0}\right)}=\frac{\eta_{j}-\left(\eta_{0}-\eta_{j}\right) t_{0}}{\left(\eta_{0}-\eta_{j}\right)-\eta_{j} t_{0}}, \quad j=1,2, \ldots, q
$$

Substitution of these last expressions into $t_{0}=t_{1} t_{2} \cdots t_{q}$ implies that $t_{0}$ is a zero of the polynomial (18).

One can ask whether $t_{0}$ is unique. The the answer is not obvious but, fortunately, we do not need it! Indeed, Lemma 3 is enough to prove Theorem 1 and the uniqueness will be numerically verified in the case of Theorem 2 .

\section{Denominators of linear forms}

In this paragraph, we prove a very general result which gives a common denominator of the coefficients of the linear forms in even beta values which are constructed in Lemma 2. Denote the least common multiple of the numbers $1,2, \ldots, n$ by $d_{n}$ and note the following consequence of the prime number theorem:

$$
\lim _{n \rightarrow \infty} d_{n}^{1 / n}=e .
$$

To find convenient denominators for the numbers (13), we split the function $R_{n}(t)$ into a product of rational functions of the form

$$
G(t)=G(a, b ; t):=\frac{(t+b)_{a-b}}{(a-b) !}=\frac{1}{\Gamma(a-b+1)} \frac{\Gamma(t+a)}{\Gamma(t+b)} \quad \text { if } a \geq b,
$$

and

$$
H(t)=H(a, b ; t):=\frac{(b-a-1) !}{(t+a)_{b-a}}=\Gamma(b-a) \frac{\Gamma(t+a)}{\Gamma(t+b)} \quad \text { if } a<b,
$$

with an integral difference $a-b$.

For $a, b \in \mathbb{Z}$, the functions (21) are integer-valued polynomials and the following property is well known (see, e.g., [Bak], Lemma 1). 
Lemma 4. Let $a, b$ be integers, $a \geq b$, and let $G(t)$ be defined in (21). Then for any non-negative integer $\lambda$, we have

$$
4^{a-b} d_{a-b}^{\lambda} \cdot D_{\lambda} G(t)_{\mid t=-k+\frac{1}{2}} \in \mathbb{Z} \quad \text { for } k \in \mathbb{Z},
$$

where the differential operator $D_{\lambda}$ is defined in (14).

The next assersion is proved in [BR], proof of Lemma 5 .

Lemma 5. Let $a, b, a_{0}, b_{0}$ be integers, $a_{0} \leq a<b \leq b_{0}$, and let $H(t)$ be defined by (22). Then for any non-negative integer $\lambda$, we have

$$
d_{b_{0}-a_{0}-1}^{\lambda} \cdot D_{\lambda}(H(t)(t+k))_{\mid t=-k} \in \mathbb{Z} \quad \text { for } k=a_{0}, a_{0}+1, \ldots, b_{0}-1 .
$$

The following result about the arithmetic of the polynomials (11) and (12) is a simple consequence of Lemmas 4 and 5. Suppose $q$ is even and the parameters $\underline{\eta}$ are ordered as follows:

$$
\eta_{1} \leq \eta_{2} \leq \cdots \leq \eta_{q-1}
$$

Set

$$
\begin{gathered}
m_{0}=\max \left\{\eta_{0}-2 \eta_{1}, \eta_{q}\right\}, \\
m_{j}=\max \left\{m_{0}, 2\left(\eta_{0}-\eta_{j}-\eta_{q}\right), 2\left(\eta_{q}-\eta_{j}\right)\right\} \quad \text { for } j=1, \ldots, q-1
\end{gathered}
$$

(obviously, $m_{1} \geq m_{2} \geq \cdots \geq m_{q-1} \geq m_{0}$ ).

Lemma 6. Under the above conditions, for $j=0,1, \ldots, q-1$,

$$
d_{m_{1} n} d_{m_{2} n} \cdots d_{m_{q-1-j} n} \cdot 16^{\eta_{q} n} \cdot P_{j n}(z) \in \mathbb{Z}[z] .
$$

Proof. Decomposing

$$
\begin{aligned}
R_{n, q, \underline{\eta}}(t)\left(t+k-\frac{1}{2}\right)^{q-1}=\left(2 t+h_{0}\right) \cdot G\left(h_{q}, 1 ; t\right) \cdot G\left(h_{0}, 1+h_{0}-h_{q} ; t\right) \\
\times \prod_{j=1}^{q-1} H\left(h_{j}, 1+h_{0}-h_{j} ; t\right)\left(t+k-\frac{1}{2}\right),
\end{aligned}
$$

and applying Lemmas 4, 5 and the Leibniz rule for differentiating a product to formulae (13), we get that

$$
d_{m_{0} n}^{q-1-j} \cdot 16^{\eta_{q} n} \cdot c_{k j} \in \mathbb{Z}
$$

for $k=h_{j}+\frac{1}{2}, \ldots, h_{0}-h_{j}+\frac{1}{2}$ and $j=1, \ldots, q-1$. On the other hand, we get

$$
\prod_{i=1}^{j} d_{\max \left\{2 h_{0}-2 h_{i}-2 h_{q}, 2 h_{q}-2 h_{i}\right\}} \cdot \sum_{l=1}^{k-h_{q}} \frac{1}{(2 l-1)^{j}} \in \mathbb{Z} .
$$

By (24) and (25), we finally arrive at the desired result (23) for the polynomials $P_{j n}(z)$ and $P_{0 n}(z)$.

Remark. Lemma 6 is not the end of our arithmetic investigations : we will slightly modify the relations (23) for Theorem 1 in the next paragraph and then sharpen them by using $p$-adic valuation of the numbers (13) for Theorem 2 in Paragraph 7. 


\section{Proof of Theorem 1}

This paragraph is devoted to the proof of Theorem 1, which is based on the following theorem of Nesterenko [Ne].

Linear independence criterion. Let $\theta_{1}, \theta_{2}, \ldots, \theta_{N}$, with $N \geq 2$, be real numbers, and assume there exist $N$ sequences of integers $\left(p_{l n}\right)_{n \geq 0}$ such that:

(i) $\alpha_{1}^{n+\mathrm{o}(n)} \leq\left|\sum_{l=1}^{N} p_{l n} \theta_{l}\right| \leq \alpha_{2}^{n+\mathrm{o}(n)}$ for certain $0<\alpha_{1} \leq \alpha_{2}<1$;

(ii) for all $l=1, \ldots, N, \quad\left|p_{l n}\right| \leq \beta^{n+o(n)}$ for certain $\beta>1$.

Then,

$$
\operatorname{dim}_{\mathbb{Q}}\left(\mathbb{Q} \theta_{1}+\mathbb{Q} \theta_{2}+\cdots+\mathbb{Q} \theta_{N}\right) \geq \frac{\log (\beta)-\log \left(\alpha_{1}\right)}{\log (\beta)-\log \left(\alpha_{1}\right)+\log \left(\alpha_{2}\right)} .
$$

In order to complete the proof, we now take the following special values for the parameters $\underline{\eta}$ :

$$
\eta_{0}=2 r+1, \quad \eta_{1}=\eta_{2}=\cdots=\eta_{q}=r, \quad \text { where } 1 \leq r \leq \frac{q-1}{2} .
$$

The new parameter $r$ is an even integer that will be chosen later as a function of $q$. As the criterion explicitly indicates, we also have to find the asymptotic behaviour of the coefficients of the linear form in this special case (26).

Lemma 7. For all $j=0,1, \ldots, q-1$,

$$
\limsup _{n \rightarrow \infty}\left|P_{j n}(-1)\right|^{1 / n} \leq 2^{q-2 r-1}(2 r+1)^{2 r+1} r^{-2 r} .
$$

We omit the proof of this lemma since it follows the same lines as [BR], Lemma 4: we use Cauchy's formula

$$
c_{k j}=\frac{1}{2 \pi i} \int_{\left|t+k-\frac{1}{2}\right|=\frac{1}{2}} R_{n}(t)\left(t+k-\frac{1}{2}\right)^{j-1} \mathrm{~d} t
$$

for the numbers (13) and then use a suitable estimation for $R_{n}(t)$ on the circle.

Lemma 8. For all $j=0,1, \ldots, q-1$,

$$
d_{2 n}^{q-1-j} \cdot 16^{r n} \cdot \frac{(r n) !^{2}}{(2 n) !^{r}} \cdot P_{j n}(z) \in \mathbb{Z}[z] .
$$


Proof. Using the decomposition

$$
\begin{aligned}
\frac{(r n) !^{2}}{(2 n) !^{r}} & \cdot R_{n}(t)\left(t+k-\frac{1}{2}\right)^{q-1} \\
= & \left(2 t+h_{0}\right) \cdot \prod_{j=1}^{r / 2} G(2 j n+1,2(j-1) n+1 ; t) \\
& \times \prod_{j=1}^{r / 2} G((r+2 j+1) n+1,(r+2 j-1) n+1 ; t) \\
& \times\left(H\left(r n+\frac{1}{2},(r+1) n+\frac{3}{2} ; t\right)\left(t+k-\frac{1}{2}\right)\right)^{q-1}
\end{aligned}
$$

for $k=r n+1, \ldots,(r+1) n+1$ and then proceeding in the same way as in the proof of Lemma 6 , we get Lemma 8.

We also need a simplified version of Lemma 3, given by the following

Lemma 9. With the above choice (26) of the parameters $\eta$, the quantity (17) satisfies

$$
0<\varphi \leq \frac{2^{2 r+1}}{r^{q-1}}
$$

Proof. By Lemma 3, $\varphi_{q, \underline{\eta}}>0$ and since $1+t_{1} t_{2} \cdots t_{q} \geq 1$ for $t_{j} \in[0,1]$, we obtain

$$
\begin{aligned}
\varphi_{q, \underline{\underline{\eta}}} & \leq \frac{(2 r+1)^{2 r+1}}{r^{2 r}} \cdot \max _{\underline{t \in[0,1]^{q}}} \prod_{j=1}^{q} t_{j}^{r}\left(1-t_{j}\right)=\frac{(2 r+1)^{2 r+1}}{r^{2 r}} \cdot\left(\max _{t \in[0,1]} t^{r}(1-t)\right)^{q} \\
& \leq \frac{(2 r+1)^{2 r+1}}{r^{2 r}} \cdot \frac{r^{q r}}{(r+1)^{q(r+1)}} \leq \frac{2^{2 r+1}}{r^{q-1}} .
\end{aligned}
$$

In order to obtain linear forms in 1 and even beta values, we assume from now on that $q$ is even (as in Lemma 2).

Define, for $j=0,1, \ldots, q / 2-1$,

$$
p_{j n}:=2^{q-2} d_{2 n}^{q-1} 16^{r n}(r n) !^{2}(2 n) !^{-r} P_{2 j, n}(-1) ;
$$

from Lemma $8, p_{j n} \in \mathbb{Z}$ for all $j$.

We also define

$$
\ell_{n}:=2^{q-2} d_{2 n}^{q-1} 16^{r n}(r n) !^{2}(2 n) !^{-r} S_{n}(-1) .
$$

According to Lemma 2,

$$
\ell_{n}=p_{0 n}+\sum_{j=1}^{q / 2-1} p_{j n} \beta(2 j)
$$


is a linear form in 1 and even beta values with integral coefficients. Stirling's formula and (20) imply that

$$
\lim _{n \rightarrow \infty}\left(2^{q-2} d_{2 n}^{q-1} 16^{r n} \frac{(r n) !^{2}}{(2 n) !^{r}}\right)^{1 / n}=e^{2(q-1)} 4^{r} r^{2 r} .
$$

From Lemma 3 we deduce that

$$
\lim _{n \rightarrow \infty}\left|\ell_{n}\right|^{1 / n}=e^{2(q-1)} 4^{r} r^{2 r} \varphi=: \kappa
$$

and from Lemma 7 that for all $j=0, \ldots, q / 2-1$,

$$
\limsup _{n \rightarrow \infty}\left|p_{j n}\right|^{1 / n} \leq e^{2(q-1)} 4^{r} 2^{q-2 r-1}(2 r+1)^{2 r+1}=: \tau \text {. }
$$

We can now apply Nesterenko's criterion to the linear form (28) with $N=$ $q / 2, \alpha_{1}=\alpha_{2}=\kappa$ and $\beta=\tau$ : we get the lower bound

$$
\begin{aligned}
\delta(q-2) & \geq \frac{(q-2 r-1) \log (2)+(2 r+1) \log (2 r+1)-2 r \log (r)-\log (\varphi)}{(2+\log (2))(q-1)+(2 r+1) \log (2 r+1)} \\
& \geq \frac{q \log (r)+(q-2 r-1) \log (2)}{(2+\log (2))(q-1)+(2 r+1) \log (2 r+1)} .
\end{aligned}
$$

In the last step, we have used Lemma 9 and the fact that $\log (2 r+1) \geq$ $\log (2 r)$. Now, as $q, r \rightarrow \infty$,

$$
q \log (r)+(q-2 r-1) \log (2)=q \log (r)+\mathrm{O}(q)+\mathrm{O}(r)=q \log (r)+\mathrm{O}(q)
$$

and

$$
(2+\log (2))(q-1)+(2 r+1) \log (2 r+1)=(2+\log (2)) q+\mathrm{O}(r \log (r)) .
$$

Thus, for $r=2\left\lfloor q / \log ^{2}(q)\right\rfloor$, we obtain

$$
\delta(q-2) \geq \frac{(1+\mathrm{o}(1)) q \log (q)}{(2+\log (2)) q+\mathrm{o}(q)}=\frac{1+\mathrm{o}(1)}{2+\log (2)} \log (q),
$$

which proves the estimate (1) of Theorem 1 . Here and in the rest of this article, $\lfloor\cdot\rfloor$ denotes the integral part of a real number. The existence of a constant $c_{0}$, for which the inequality $\delta(a)>c_{0} \log (a)$ always holds, immediately follows from the absolute lower bound (29) for the above choice of $r$. 


\section{$7 \quad p$-adic valuation of the coefficients}

Since $\operatorname{ord}_{p} d_{N}=1$ for those primes $p$ such that $\sqrt{N}<p \leq N$, Lemma 6 gives that the $p$-adic order of the coefficients of the polynomials $P_{j n}(z)$ and $P_{0 n}(z)$ is at least $-(q-1)$ if $\sqrt{m_{1} n}<p \leq m_{q-1} n$. The aim of this paragraph is to sharpen these lower bounds for $p$-adic orders.

The next assertion is proved in [Zu2], Lemma 18.

Lemma 10. Let $a, b, a_{0}, b_{0}$ be integers, $a_{0} \leq a<b \leq b_{0}$, and let $H(t)=$ $H(a, b ; t)$ be defined as in (22). Then for any non-negative integer $j$, any integer $k, a_{0} \leq k<b_{0}$, and any prime $p>\sqrt{b_{0}-a_{0}-1}$, the following holds

$$
\operatorname{ord}_{p}(H(t)(t+k))_{\mid t=-k}^{(j)} \geq-j+\left\lfloor\frac{b-a-1}{p}\right\rfloor-\left\lfloor\frac{k-a}{p}\right\rfloor-\left\lfloor\frac{b-1-k}{p}\right\rfloor .
$$

The following observation will be useful later on: $\operatorname{ord}_{p} N !=\lfloor N / p\rfloor$ for $p>\sqrt{N}$. An easy consequence of this fact is the formula giving the $p$-adic order of the numbers

$$
\frac{\Gamma(N+1 / 2)}{\Gamma(1 / 2)}=\left(\frac{1}{2}\right)_{N}=4^{-N} \frac{(2 N) !}{N !}
$$

which are sometimes written as $(2 N) ! ! \cdot 2^{-N}$; namely,

$$
\operatorname{ord}_{p}(2 N) ! !=\llbracket \frac{N}{p} \| \quad \text { for } p>\sqrt{2 N}, p \neq 2,
$$

where $\lfloor x\rfloor:=\lfloor 2 x\rfloor-\lfloor x\rfloor$.

Lemma 11. Let $a, b, a_{0}, b_{0}$ be integers, $b_{0} \leq b<a \leq a_{0}$, and let $G(t)=$ $G(a, b ; t)$ be defined as in (21). Then for any non-negative integer $j$, any integer $k, b_{0}<k<a_{0}$, and any prime $p>\sqrt{2\left(a_{0}-b_{0}-1\right)}, p \neq 2$, the following holds :

$$
\begin{aligned}
\operatorname{ord}_{p} G^{(j)}\left(-k+\frac{1}{2}\right) & \left.\geq-j+\left\|\frac{a-k}{p}\right\|-\| \frac{b-k}{p}\right\rfloor-\left\lfloor\frac{a-b}{p}\right\rfloor \\
& \left.=-j-\llbracket \frac{k-a}{p}\right\rfloor+\left\lfloor\frac{k-b}{p}\right\rfloor-\left\lfloor\frac{a-b}{p}\right\rfloor .
\end{aligned}
$$

Proof. By (30) we deduce that

$$
4^{a-b} G\left(-k+\frac{1}{2}\right)= \begin{cases}\frac{(2 a-2 k) !(b-k) !}{(a-k) !(2 b-2 k) !(a-b) !} & \text { if } k \leq b, \\ (-1)^{k-b} \frac{(2 k-2 b) !(2 a-2 k) !}{(k-b) !(a-k) !(a-b) !} & \text { if } b<k<a, \\ (-1)^{a-b} \frac{(2 k-2 b) !(k-a) !}{(k-b) !(2 k-2 a) !(a-b) !} & \text { if } k \geq a .\end{cases}
$$


Using (31) and the formula $\llbracket-N / p \rrbracket=-\llbracket N / p \rrbracket$ (for integers $N$ and primes $p>2$ ), we obtain the desired estimates (32) for $j=0$. Inductive arguments, similar to that given in the proof of Lemma 17 in [Zu2], prove (32) for all $j$.

Applying Lemmas 10, 11 and the Leibniz rule (for differentiating a product) to the decomposition

$$
\begin{aligned}
R_{n, q, \underline{\eta}}(t)=(2 t & \left.+h_{0}\right) \cdot G\left(h_{q}, 1 ; t\right) \cdot G\left(h_{0}, 1+h_{0}-h_{q} ; t\right) \\
& \times \prod_{j=1}^{q-1} H\left(h_{j}, 1+h_{0}-h_{j} ; t\right)
\end{aligned}
$$

gives rise to the estimations

$$
\begin{aligned}
\operatorname{ord}_{p} D_{\lambda}\left(R_{n}(t)\left(t+k-\frac{1}{2}\right)^{q-1}\right)_{\mid t=-k+\frac{1}{2}} & \geq-\lambda+\varpi_{0}\left(\frac{n}{p}, \frac{k-1}{p}\right) \\
& \geq-\lambda+\varpi\left(\frac{n}{p}\right)
\end{aligned}
$$

for $p>\max \left\{\sqrt{2 \eta_{0} n}, q-1\right\}, \lambda=0,1, \ldots, q-1$, and $k=h_{1}+\frac{1}{2}, \ldots, h_{0}-h_{1}+\frac{1}{2}$, where

$$
\begin{aligned}
\varpi_{0}(x, y):= & \sum_{j=1}^{q-1}\left(\left\lfloor\left(\eta_{0}-2 \eta_{j}\right) x\right\rfloor-\left\lfloor y-\eta_{j} x\right\rfloor-\left\lfloor\left(\eta_{0}-\eta_{j}\right) x-y\right\rfloor\right) \\
& +\left\lfloor\eta_{0} x-y \Perp-\left\lfloor y-\eta_{q} x \Perp+\lfloor y\rfloor-\left\lfloor\left(\eta_{0}-\eta_{q}\right) x-y \bigsqcup-2\left\lfloor\eta_{q} x\right\rfloor,\right.\right.\right. \\
\varpi(x):= & \min _{y \in \mathbb{R}} \varpi_{0}(x, y)=\min _{0 \leq y<1} \varpi_{0}(x, y) .
\end{aligned}
$$

The function $\varpi(x)$ is non-negative, 1-periodic and integer-valued. Setting

$$
\Pi_{n}:=\prod_{\sqrt{2 \eta_{0} n}<p \leq m_{q-1} n} p^{\varpi(n / p)}
$$

and recalling the formula (13), we finally obtain the following sharpening of Lemma 6.

Lemma 12. For $j=0,1, \ldots, q-1$, the following hold

$$
d_{m_{1} n} d_{m_{2} n} \cdots d_{m_{q-1} n} \cdot 16^{\eta_{q} n} \cdot \Pi_{n}^{-1} \cdot P_{j n}(z) \in \mathbb{Z}[z] .
$$

The asymptotic behaviour of (33) as $n \rightarrow \infty$ is given by (20); we refer the reader to [Ha] and [Zu1], Lemma 4.4, for details.

Lemma 13. In the limit when $n \rightarrow+\infty$,

$$
\lim _{n \rightarrow \infty} \frac{\log \Pi_{n}}{n}=\int_{0}^{1} \varpi(x) \mathrm{d} \psi(x)-\int_{0}^{1 / m_{q-1}} \varpi(x) \frac{\mathrm{d} x}{x^{2}},
$$

where $\psi(x)=\Gamma^{\prime}(x) / \Gamma(x)$. 


\section{Proof of Theorem 2}

Unlike Theorem 1, proving Theorem 2 only requires the following

Irrationality criterion. Let $\theta_{1}, \ldots, \theta_{N}$ be real numbers. Assume there exists $N$ sequences of integers $\left(p_{l n}\right)_{n \geq 0}$ such that the linear form $p_{1 n} \theta_{1}+p_{2 n} \theta_{2}+$ $\cdots+p_{N n} \theta_{N}$ is non-zero for infinitely many $n$ and tends to 0 as $n \rightarrow \infty$. Then at least one of the numbers $\theta_{1}, \ldots, \theta_{N}$ is irrational.

This clearly follows from Nesterenko's criterion but in this case the proof is completely elementary. Note also that here we do not need an estimation of the coefficients of the linear form.

Taking $q=16$ and

$$
\underline{\eta}=(101,28,29,30,31,32,33,34,35,36,37,38,39,40,41,42,45),
$$

we obtain $t_{0} \approx 0.00005445875955$. In this case, it is the unique zero in $[0,1]$ of $Q(t)$; Further, $\varphi_{q, \eta} \approx \exp (-594.58616762)$ and

$$
\lim _{n \rightarrow \infty} \frac{\log \left(d_{m_{1} n} d_{m_{2} n} \cdots d_{m_{q-1} n} \cdot 16^{\eta_{q} n} \cdot \Pi_{n}^{-1}\right)}{n} \approx 593.98582857
$$

since

$$
\begin{gathered}
m_{1}=56, m_{2}=54, m_{3}=52, m_{4}=50, m_{5}=48, m_{6}=46, \\
m_{7}=\cdots=m_{15}=45,
\end{gathered}
$$

and the function $\varpi(x)$ takes values in $\{0,1, \ldots, 9\}$. Thus, the irrationality criterion and Lemma 2 yield that at least one of the numbers (2) is irrational.

\section{Special emphasis on Catalan's constant}

When $q=4$, our general construction gives us linear forms in 1 and Catalan's constant $G=\beta(2)$, and the corresponding well-poised hypergeometric series (5) for $z=-1$ can be reduced to a simpler series using Bailey's identity [Bai], Section 4.4, formula (2) :

$$
\begin{aligned}
& { }_{3} F_{2}\left(\begin{array}{c|c}
1+a-b-c, d, e \\
1+a-b, 1+a-c
\end{array}\right)=\frac{\Gamma(1+a) \Gamma(1+a-d-e)}{\Gamma(1+a-d) \Gamma(1+a-e)} \\
& \times{ }_{6} F_{5}\left(\begin{array}{c}
6 a, 1+\frac{1}{2} a, \quad b, \quad c, \quad c, \quad e \\
\frac{1}{2} a, \quad 1+a-b, 1+a-c, 1+a-d, 1+a-e
\end{array} \mid-1\right),
\end{aligned}
$$


if $\operatorname{Re}(1+a-d-e)>0$. We obtain

$$
\begin{aligned}
s_{n, \underline{\eta}}:= & S_{n, 4, \underline{\eta}}(-1) \\
= & \frac{\Gamma\left(h_{1}\right) \Gamma\left(h_{2}\right) \Gamma\left(h_{3}\right) \Gamma\left(1+h_{0}-2 h_{1}\right) \Gamma\left(1+h_{0}-2 h_{2}\right) \Gamma\left(1+h_{0}-2 h_{3}\right)}{\Gamma\left(1+h_{0}-h_{1}\right) \Gamma\left(1+h_{0}-h_{2}\right) \Gamma\left(1+h_{0}-h_{3}-h_{4}\right) \Gamma\left(h_{4}\right)} \\
& \quad \times{ }_{3} F_{2}\left(\begin{array}{l}
1+h_{0}-h_{1}-h_{2}, h_{3}, h_{4} \\
1+h_{0}-h_{1}, 1+h_{0}-h_{2}
\end{array}\right) \\
= & \frac{\Gamma\left(h_{1}\right) \Gamma\left(h_{2}\right) \Gamma\left(1+h_{0}-2 h_{1}\right) \Gamma\left(1+h_{0}-2 h_{2}\right) \Gamma\left(1+h_{0}-2 h_{3}\right)}{\Gamma\left(1+h_{0}-h_{1}-h_{3}\right) \Gamma\left(1+h_{0}-h_{2}-h_{4}\right) \Gamma\left(1+h_{0}-h_{3}-h_{4}\right) \Gamma\left(h_{4}\right)^{2}} \\
& \quad \times \int_{[0,1]^{2}} \frac{x^{h_{3}-1}(1-x)^{h_{0}-h_{1}-h_{3}} y^{h_{4}-1}(1-y)^{h_{0}-h_{2}-h_{4}}}{(1-x y)^{h_{0}-h_{1}-h_{2}}} \frac{\mathrm{d} x y}{1-x y} .
\end{aligned}
$$

Since the ${ }_{3} F_{2}$-series in (34) is invariant under any permutation of its top or bottom parameters, we deduce that the permutation of its top integral parameters $1+h_{0}-h_{1}-h_{2}$ and $h_{4}$ produces the transformation

$$
\mathfrak{b}: \underline{\eta} \mapsto\left(2 \eta_{0}-\eta_{1}-\eta_{2}-\eta_{4}, \eta_{0}-\eta_{2}-\eta_{4}, \eta_{0}-\eta_{1}-\eta_{4}, \eta_{3}, \eta_{0}-\eta_{1}-\eta_{2}\right)
$$

under which

$$
\frac{s_{n, \underline{\eta}}}{\prod_{j=1}^{3} \Gamma\left(\eta_{j} n+\frac{1}{2}\right) \Gamma\left(1+\left(\eta_{0}-2 \eta_{j}\right) n\right) \Gamma\left(1+\left(\eta_{0}-\eta_{1}-\eta_{2}-\eta_{3}+\eta_{j}\right) n\right)}
$$

is invariant. We note that (36) is also stable under permutation of the nonintegral parameters $h_{1}, h_{2}, h_{3}$ of the original well-poised ${ }_{6} F_{5}$-series. They are $6=3$ ! such permutations and we can consider the group $\mathfrak{G}=\left\langle\mathfrak{b}, \mathfrak{S}_{3}\right\rangle$ of the transformations of the parameters $\eta$ that acts trivially on (36). This group has order 24 and is a subgroup of the permutation group of order 120 discovered by Rhin and Viola [RV] for $\zeta(2)$ (where permutations of $h_{1}, h_{2}, h_{3}$, and $h_{4}$ are allowed, cf. [Zu2], Section 6). Unfortunately, the 'denominators' of the linear forms $s_{n, \eta}$ given by Lemma 6 are too large to be able to use the arithmetic arguments in [RV], [Zu2].

Finally, we want to mention an arithmetic observation for linear forms in the case $\eta_{0}=3, \eta_{1}=\eta_{2}=\eta_{3}=\eta_{4}=1$ :

$$
s_{n, \underline{\eta}}=\int_{[0,1]^{2}} \frac{x^{n-\frac{1}{2}}(1-x)^{n} y^{n}(1-y)^{n-\frac{1}{2}}}{(1-x y)^{n+1}} \mathrm{~d} x \mathrm{~d} y \in \mathbb{Q} G+\mathbb{Q} .
$$

Thanks to Zeilberger's creative telescoping algorithm, we have found (see [Zu4]) that both the sequence $(-1)^{n} s_{n, \underline{\eta}}=u_{n} G-v_{n}$ and the corresponding coefficients $u_{n}$ and $v_{n}$ satisfy the Apéry-like difference equation

$$
(2 n+1)^{2}(2 n+2)^{2} p(n) u_{n+1}-q(n) u_{n}-(2 n-1)^{2}(2 n)^{2} p(n+1) u_{n-1}=0,
$$


where

$$
\begin{aligned}
& p(n)=20 n^{2}-8 n+1, \\
& q(n)=3520 n^{6}+5632 n^{5}+2064 n^{4}-384 n^{3}-156 n^{2}+16 n+7,
\end{aligned}
$$

with the initial data

$$
u_{0}=4, \quad u_{1}=7, \quad v_{0}=0, \quad v_{1}=\frac{13}{2} .
$$

Using the recursion (37), we have verified numerically (up to $n=1000$ ) that $16^{n} \cdot u_{n} \in \mathbb{Z}$ and $d_{2 n}^{2} \cdot 16^{n} \cdot v_{n} \in \mathbb{Z}:$ this is more that one can get from Lemma 6 , although it is not sufficient to prove the irrationality of Catalan's constant.

Acknowledgements. We would like to express our gratitude to S. Fischler, M. Waldschmidt and the anonymous referee for their helpful remarks which have much improved this article.

\section{References}

[Ap] R. Apéry, Irrationalité de $\zeta(2)$ et $\zeta(3)$, Astérisque 61, 11-13 (1979)

[Bai] W. N. Bailey, Generalized hypergeometric series, Cambridge Math. Tracts 32 (Cambridge University Press, Cambridge 1935); 2nd reprinted edition (Stechert-Hafner, New York 1964)

[Bak] A. Baker, The theory of linear forms in logarithms, Transcendence theory: advances and applications (Proc. Conf., Univ. Cambridge, Cambridge, 1976), pp. 1-27 (Academic Press, London, 1977)

[BR] K. Ball, T. Rivoal, Irrationalité d'une infinité de valeurs de la fonction zêta aux entiers impairs, Invent. Math. 146, no. 1, 193-207 (2001)

[Ha] M. Hata, Legendre type polynomials and irrationality measures, J. Reine Angew. Math. 407, no. 1, 99-125 (1990)

[Ne] Yu. V. Nesterenko, On the linear independence of numbers, Vestnik Moskov. Univ. Ser. I Mat. Mekh. no. 1, $46-54$ (1985); English transl., Moscow Univ. Math. Bull. 40, no. 1, 69-74 (1985)

[RV] G. Rhin, C. Viola, On a permutation group related to $\zeta(2)$, Acta Arith. 77, no. 1, 23-56 (1996) 
[Ri1] T. Rivoal, La fonction zêta de Riemann prend une infinité de valeurs irrationnelles aux entiers impairs, C. R. Acad. Sci. Paris Sér. I Math. 331, no. 4, 267-270 (2000)

[Ri2] T. Rivoal, Irrationalité d'au moins un des neuf nombres $\zeta(5), \zeta(7), \ldots, \zeta(21)$, Acta Arith. 103, 157-167 (2002)

[Sl] L.J. Slater, Generalized hypergeometric functions, 2nd edition (Cambridge University Press, Cambridge 1966)

[Zu1] W. Zudilin, Irrationality of values of Riemann's zeta function, Izv. Ross. Akad. Nauk Ser. Mat. 66, no. 3, 49-102 (2002); English transl., Russian Acad. Sci. Izv. Math. 66, no. 3 (2002)

[Zu2] W. Zudilin, Arithmetic of linear forms involving odd zeta values, Preprint (August 2001); E-print math.NT/0206176, 42 pages (2002)

[Zu3] V.V. Zudilin, One of the numbers $\zeta(5), \zeta(7), \zeta(9), \zeta(11)$ is irrational, Uspekhi Mat. Nauk 56, no. 4, 149-150 (2001); English transl., Russian Math. Surveys 56, no. 4, 774-776 (2001)

[Zu4] W. Zudilin, Apéry-like difference equation for Catalan's constant, E-print math.NT/0201024, 10 pages (2002)

Tanguy Rivoal

Institut de Mathématiques de Jussieu

CNRS UMR 7586, Théorie des Nombres, case 247

175, rue du Chevaleret

75013 Paris, FRANCE

email : rivoal@math.jussieu.fr

Wadim Zudilin

Moscow Lomonosov State University

Department of Mechanics and Mathematics

Vorobiovy Gory, GSP-2

119992 Moscow, RUSSIA

email : wadim@ips.ras.ru 